\title{
Simultaneously Measuring Red Blood Cell Flux in vivo for a Large Number of Retinal Capillary Vessels Using Optical Coherence Tomography
}

\author{
Ruikang K. Wang ${ }^{1,2}$, and Zhongwei $\mathrm{Zhi}^{1}$ \\ 1. Department of Bioengineering, University of Washington, Seattle, WA 98195, USA \\ 2. Department of Ophthalmology, University of Washington, Seattle, WA 98195, USA
}

In this presentation, we will present an application of optical coherence tomography (OCT) imaging technique for measurement of red blood cell (RBC) flux within multiple capillary of retina in mice in vivo. The technique would be also useful in the monitoring of changes in RBC flux as a function of pharmacological treatment doses for diseases such as diabetes, infectious and inflammatory diseases and cancer, where monitoring vascular transport could be essential.

Ocular microcirculation plays an important role for maintaining a normal vision and abnormal ocular blood flow was shown to be related to many ocular diseases such as age-related macular degeneration, diabetic retinopathy, and glaucoma. Capillaries are the smallest unit of circulatory system, within which, red blood cells (RBCs) are squeezing through one by one. Capillary RBC flux, i.e. the number of RBC flow through the capillary per unit time, is an important capillary flow parameter. It is critical to develop a noninvasive imaging technique to visualize the retinal capillary network and quantify the RBC characteristics for understanding and early diagnosis of ocular diseases that have a vascular component in their pathogenesis. Optical coherence tomography (OCT) is a non-contact and non-invasive imaging modality and has been widely used for human eye imaging and blood flow measurement. By using a repeated B-scan mode, the fluctuating OCT signal has been shown to be associated with the RBC passing through the capillary in the mouse brain [1]. Ren et. al. also applied OCT to measure the red blood cell velocities of cerebrovascular networks by detecting the Doppler phase transients induced by the passage of RBCs through the capillary [2]. However, both of these works were done on cerebrovascular network, and no work has yet been done on retinal capillary flux measurement using OCT.

In our work, we developed a technique that used OCT imaging [3] for measurement of RBC flux within multiple capillary of retina in the mice and rat. A fast speed spectral-domain OCT imaging system at $820 \mathrm{~nm}$ with a line scan rate of $240 \mathrm{kHz}$ was developed to image mouse retina. By acquiring repeated Bscans at an ultrafast $720 \mathrm{fps}$ frame rate, we were able to detect the fluctuating OCT signal intensity caused by the passage of single RBC through the capillary (Fig.2A-C). RBC flux of each single capillary was obtained by counting the number of peaks in the OCT intensity profile after applying Gaussian filtering (Fig.2 D). Multiple capillaries in one cross-section were isolated and their flux was measured automatically with this algorithm (Fig.2 E). This developed technique was applied to measure the RBC flux of capillaries within retina of BTBR ob/ob mice, as well as wild type (WT) controls. The results (Fig. 3) indicate that the ob/ob mice retina have a lower average RBC flux than WT mice, which may provide a way for early diagnosis of Diabetic retinopathy.

In summary, we demonstrated the capability of OCT imaging technique for the measurement of RBC flux within multiple capillary of retina at the same time in mice. Fast frame rate (720 fps in this application) repeated B-scans at one cross-section enable us to observe fluctuation in OCT intensity as RBC passing through the capillary. The flux of each single capillary was estimated by counting the number of peaks per unit time in the signal profile after applying a Gaussian filtering algorithm. The 
average capillary flux within retinas of BTBR ob/ob mice were measured to statistically lower than its WT controls. This indicates that capillary RBC flux can be an important potential maker for the early detection of diabetic retinopathy as well as other ocular diseases.

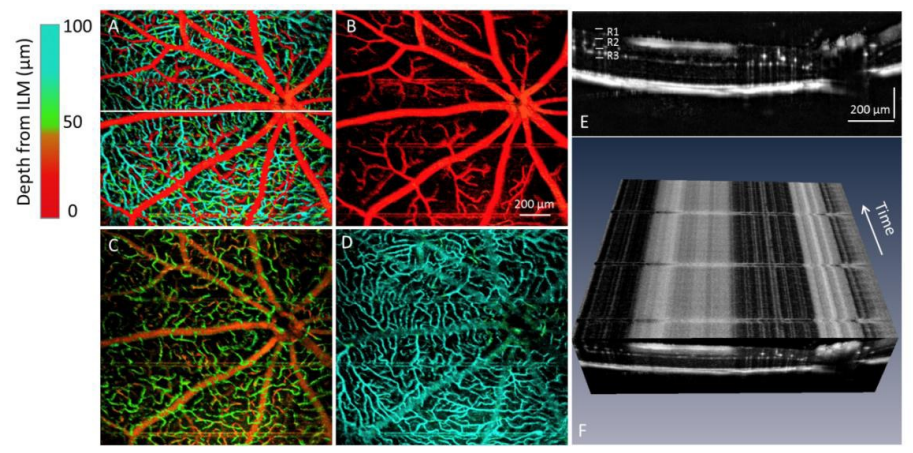

Fig. 1. (A) Depth-resolved angiography of moue retina. (B-D) Microvasculature within three different layers of retina after segmentation, where R1, R2 and R3 represent NFL/GCL, IPL, and OPL, respectively. (E) Cross-sectional blood flow image located at the white line in A. (F) Blood vessel through time obtained with repeated B-scans. The horizontal line artifacts are caused by animal breathing.
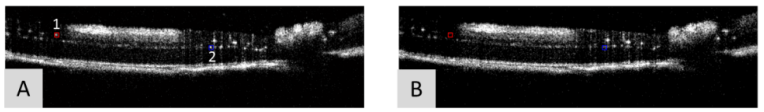

Fig. 2. (A-B) Two cross-sectional blood flow frames captured at different times showing the fluctuating capillary signal. (C) Smoothed intensity profile at the two capillary regions labeled as 1 and 2. (D) The intensity profile after Gaussian fitting, and capillary flux was calculated by detecting the number of peaks. (E) Color coded capillary flux image and (F) histogram of the flux for all capillaries in the crosssection.
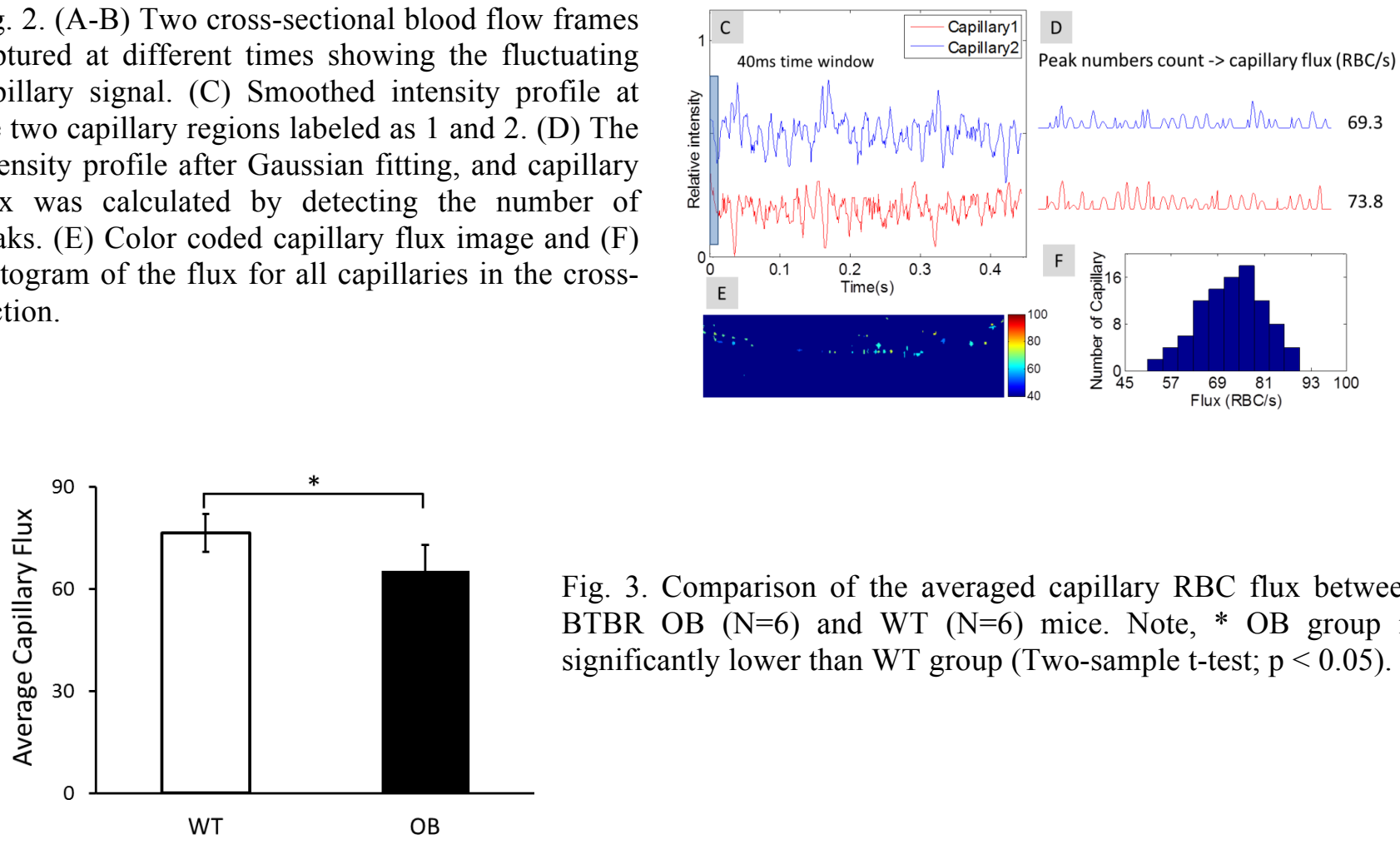

Fig. 3. Comparison of the averaged capillary RBC flux between BTBR OB $(\mathrm{N}=6)$ and WT $(\mathrm{N}=6)$ mice. Note, * OB group is significantly lower than WT group (Two-sample t-test; $\mathrm{p}<0.05$ ).

\section{References:}

1. Lee, J., et al., Journal of Cerebral Blood Flow and Metabolism, 2013. 33(11): 1707-1710.

2. Ren, H.G., et al. Applied Physics Letters, 2012. 100(23).

3. Zhi, Z., et al., Investigative Ophthalmology \& Visual Science, 2014. 55(2), 1024-1030. 